\title{
Biotherapies in Solid Tumors: Are Negative Results Still of Low Priority for Publication?
}

\begin{abstract}
Alessandro Ottaiano, Antonino Cassata, Monica Capozzi, Chiara De Divitiis, Alfonso De Stefano and Antonio Avallone*
\end{abstract}

Department of Abdominal Oncology, Istituto Nazionale Tumori Fondazione G. Pascale (IRCCS), Naples, Italy

In 2005, we performed the largest survey on clinical trials of biotherapies for all solid tumors and found indirect evidence of a publication bias: editors of medical journals were more prone to publish positive results independently from the quality of the studies. We collected data from 2003 to 2015 in 487 studies, and the publication bias previously described was not found in the years between 2010 and 2015: this could be related to changes and/or innovations in the guidelines and editorial policies of oncology journals occurred over the last years.

Keywords: biotherapies, publication bias, solid tumors, survey, methodology

\section{OPEN ACCESS}

Edited by:

Giuseppe Di Lorenzo,

Azienda Ospedaliera Universitaria

Federico II, Italy

Reviewed by:

Antonio Rozzi,

INI, Istituto Neurotraumatologico Italiano, Italy

Lello Piccirillo,

Ospedale Filippo Del Ponte

Varese, Italy

${ }^{*}$ Correspondence:

Antonio Avallone

a.avallone@istitutotumori.na.it

Specialty section:

This article was submitted to Cancer Molecular Targets and Therapeutics,

a section of the journal

Frontiers in Oncology

Received: 02 February 2018 Accepted: 23 February 2018

Published: 14 March 2018

Citation:

Ottaiano A, Cassata A, Capozzi M, De Divitiis C, De Stefano A and Avallone A (2018) Biotherapies in Solid Tumors: Are Negative Results Still of Low Priority for Publication?

Front. Oncol. 8:62.

doi: 10.3389/fonc.2018.00062
In 2005, we performed the largest survey on clinical trials of biologic and immunologic therapies (i.e., Biotherapy: inhibitors of the proliferative signals, antiangiogenic agents, differentiating agents, inhibitors of metastasis/invasiveness, drugs with multiple mechanisms of action; Immunotherapy: vaccines, cytokines, adoptive immunotherapy, antibodies stimulating the immune system, drugs with multiple immunologic mechanisms of action) for all solid tumors in order to review their content and quality (1); we found an indirect evidence of publication bias (2) as an attitude of high-impact factor medical journals to prefer the publication of positive results. We showed that publication was related to positive results independently from study quality; the observation period ranged from 1990 to 2002. This kind of publication bias (related to the reporting of positive outcome) suggested that negative results had a lower chance of being published, particularly in high-impact factor journals.

In non-oncologic research areas, there are direct and indirect pieces of evidence that this type of publication bias is increasing (3-5). This phenomenon might reduce the visibility of negative results and affect the clinical development of a drug (identification of clinical endpoints, selection of patients and therapies, management of toxicities) because this process depends on the interaction between positive and negative findings. Furthermore, oncologic research may be affected by other publication bias previously described (6-8).

Since then, we continued to collect data about biotherapies in solid tumors (general study characteristics, patient and disease factors, study methodology and quality) from selected oncology journals limiting our search to phase II/III clinical trials in colorectal, lung, breast, prostate cancer, pancreas, and melanoma from 2003 to 2015 through Medline searching. Four hundred eighty-seven clinical trials were identified. Quality of studies (quality index: QI) was determined through a modified ad hoc questionnaire (Table 1). Study evaluation methods were similar to the previous study (1); however, the impact factor was arbitrarily divided into three categories: $<6,6-10,>10$. A QI $<50$ points was calculated for $36.7 \%$ of phase II and $28.2 \%$ for phase III or II/III studies. Results were defined positive when the authors showed significant improvements in activity and/or efficacy of a new treatment, negative when it was inactive or toxic or failed to show significant ameliorations vs the referral drugs.

Here, we report on the association between quality, conclusions, and other explanatory variables of the studies and impact factor of the journals in recent years. In particular, the association between positive outcome and publication in high-impact factor journals was not found in the 
TABLE 1 | Quality assessment questionnaire for phase II and III studies.

\section{A. Patient selection}

Zero points if patients are not selected on a validated biological/molecular basis; 10 points if they are

\section{B. Trial size}

Phase II: 2 points if total number of patients is $<30 ; 6$ points if $>30$ or $<50 ; 10$ points if $>50$

Phase III: 2 points if total number of patients is $<100$; 6 points if $>100$ or $<300$;

10 points if $>300$

Analysis and measurement of biological effect

C. Study design

Zero points if not reported; 10 points if yes

D. Biological endpoints

Zero points if not reported; 10 points if evaluations of biological endpoints are planned

\section{E. Response}

Zero points if criteria for response assessment are not reported; 10 points if yes

F. Toxicity

Zero points if criteria for toxicity evaluation are not reported; 10 points if yes

G. PERI ${ }^{\mathrm{a}}$

Zero points if preclinical evidence is not cited; 2 points if $P E \mid<15 ; 8$ points if

PEI>15

\section{H. Sites of disease}

Zero points if not reported; 8 points if reported

I. Compliance to treatment

Zero points if not reported; 8 points if reported

J. Time-to-event descriptions

Eight points if overall-survival and/or disease-free survival are reported

K. Follow-up

Eight points if any information about follow-up is reported (duration and lost to follow-up)

${ }^{a}$ PERI (Preclinical Evidence Reporting Index) is the sum of the impact factors of the journals cited by the authors when discussing the preclinical background.

studies published between 2010 and 2015 (202 studies). In a multiple regression model performed to analyze the correlation between impact factor and some different explanatory variables of studies [quality ( $<50$ vs $\geq 50$ ), phase of the study (II vs III), academic/company supported (yes vs no), accrual time ( $\leq 3$ years vs $>3$ years), study conclusions (positive vs negative)] the only variable found to be predictive of publication in a high-impact factor journal between 2010 and 2015 was the quality of the study $(P=0.012)$ and not the positive result $(P=0.178)$ (Table 2$)$.

\section{REFERENCES}

1. Ottaiano A, Mollo E, Di Lorenzo G, Pisano C, Di Maio M, Barletta E, et al. Prospective clinical trials of biotherapies in solid tumors: a 5-year survey. Cancer Immunol Immunother (2005) 54:44-50. doi:10.1007/s00262004-0567-z

2. Ottaiano A, Castello G, Ascierto PA. Evidence of publication bias in clinical trials of biotherapies for solid tumors. Cancer (2005) 103:653. doi:10.1002/ cncr.20835

3. Easterbrook PJ, Berlin JA, Gopalan R, Matthews DR. Publication bias in clinical research. Lancet (1991) 337:867-72. doi:10.1016/0140-6736(91)90201-Y

4. Kjaergard LL, Als-Nielsen B. Association between competing interests and authors' conclusions: epidemiological study of randomised clinical trials published in the BMJ. BMJ (2002) 325:249. doi:10.1136/bmj.325.7358.249

5. Kicinski M. Publication bias in recent meta-analyses. PLoS One (2013) 8:e81823. doi:10.1371/journal.pone.0081823. eCollection 2013. Erratum in: PLoS One (2014) 9. doi:10.1371/annotation/a65c0f61-eb99-42f0-828b-5a8662bce4f7

6. Ramsey S, Scoggins J. Commentary: practicing on the tip of an information iceberg? Evidence of underpublication of registered clinical trials in oncology. Oncologist (2008) 13:925-9. doi:10.1634/theoncologist.2008-0133
TABLE 2 | Multiple regression to analyze the relationship between explanatory variables of studies and publication in high-impact factor journals between 2010 and 2015 .

\begin{tabular}{llcccc}
\hline Variable & No. of studies & Coefficient & SE & $\boldsymbol{R}_{\text {partial }}$ & $\boldsymbol{P}$ \\
\hline Quality & $<50: 50 ;>50: 152$ & 0.72 & 0.25 & 0.68 & 0.012 \\
Company driven & Yes: 121; No: 81 & 0.36 & 0.11 & 0.14 & 0.081 \\
Accrual time & $<$ 3 years: 158; & 0.28 & 0.16 & 0.19 & 0.172 \\
& $>$ 3 years: 44 & & & & \\
Study conclusion & Positive: 137; & 0.45 & 0.19 & 0.15 & 0.226 \\
& Negative: 65 & & & & \\
Phase & II: 142; III or II/III: 60 & 0.33 & 0.21 & 0.09 & 0.198 \\
\hline
\end{tabular}

One possible explanation of this specific outcome-related publication bias reduction may be related to changes and/or innovations in the guidelines and editorial policies (reception, evaluation, and publication of studies with negative results) of oncology journals occurred over the last years. Our report has some limitations: (i) it represents an indirect evidence about publication bias since the "true publication bias" is the difference between published and "unpublished" studies (i.e., discordance between trial in official registries and published trial) and (ii) our QI has not been independently validated by other research groups despite being reasonably a useful tool to resume the most important methodology characteristics of a clinical study.

New generation targeted therapies are changing the therapeutic management of advanced tumors and the interest on studying new therapeutic pathways as well as new drugs is increasing. We show an indirect evidence that editors and reviewers have improved in past few years their overall methodology when discussing and evaluating clinical studies on biotherapies.

\section{AUTHOR CONTRIBUTIONS}

$\mathrm{AO}$ contributed in planning, analysis, discussing, and writing of this manuscript. AC and MC contributed in discussing and writing of the manuscript. CD and AS contributed in analysis and writing of the manuscript. AA contributed in discussing of the manuscript.

7. Takeda A, Loveman E, Harris P, Hartwell D, Welch K. Time to full publication of studies of anti-cancer medicines for breast cancer and the potential for publication bias: a short systematic review. Health Technol Assess (2008) 12:iii, ix-X, 1-46. doi:10.3310/hta12320

8. Peppercorn J, Blood E, Winer E, Partridge A. Association between pharmaceutical involvement and outcomes in breast cancer clinical trials. Cancer (2007) 109:1239-46. doi:10.1002/cncr.22528

Conflict of Interest Statement: The authors declare that the research was conducted in the absence of any commercial or financial relationships that could be construed as a potential conflict of interest.

Copyright (C) 2018 Ottaiano, Cassata, Capozzi, De Divitiis, De Stefano and Avallone. This is an open-access article distributed under the terms of the Creative Commons Attribution License (CC BY). The use, distribution or reproduction in other forums is permitted, provided the original author(s) and the copyright owner are credited and that the original publication in this journal is cited, in accordance with accepted academic practice. No use, distribution or reproduction is permitted which does not comply with these terms. 